\title{
Après le mur : les représentations israéliennes de la séparation avec les Palestiniens
}

After the wall: Israeli representations of the separation with Palestinians

\section{Cédric Parizot}

\section{(2) OpenEdition}

12 Journals

\section{Édition électronique}

URL : http://journals.openedition.org/conflits/17532

DOI : $10.4000 /$ conflits. 17532

ISSN : $1777-5345$

Éditeur :

CCLS - Centre d'études sur les conflits lilberté et sécurité, L'Harmattan

\section{Édition imprimée}

Date de publication : 30 mars 2009

Pagination : 53-72

ISBN : 978-2-296-07748-5

ISSN : 1157-996X

Référence électronique

Cédric Parizot, «Après le mur : les représentations israéliennes de la séparation avec les

Palestiniens », Cultures \& Conflits [En ligne], 73 | printemps 2009, mis en ligne le 30 mars 2010,

consulté le 30 mars 2021. URL : http://journals.openedition.org/conflits/17532 ; DOI : https://doi.org/ $10.4000 /$ conflits. 17532 


\title{
Après le mur : les représentations israéliennes de la séparation avec les Palestiniens
}

\section{Cédric PARIZOT}

Cédric Parizot est chercheur an CNRS et an Centre de recherche français de Jérusalem. Ses recherches portent d'une part sur les mobilités et les échanges transfrontaliers dans l'espace israélo-palestinien et, d'autre part, sur les processus électoraux chez les Palestiniens d'Israël.

\begin{abstract}
A'été 2002, le gouvernement d'Ariel Sharon lance la construction d'une $\mathrm{A}_{\text {«barrière de sécurité » (gader bitahon })^{1} \text { autour de la Cisjordanie. Prévue }}$ pour s'étendre sur près de $723 \mathrm{~km}$ de long, cette structure vient consolider physiquement le régime de séparation que les Israéliens ont imposé aux Palestiniens depuis la période d'Oslo (1993-2000) 22. Elle est alors présentée comme une solution radicale au conflit. L'érection de murs de bétons et de grillages doit fournir une parade décisive contre les attentats-suicides palestiniens en territoire israélien. Elle doit, ensuite, restaurer les limites souveraines de l'Etat d'Israël. En 2005, trois ans après son lancement, les attentats ont considérablement diminué et les ouvriers palestiniens qui travaillaient en Israël semblent avoir complètement disparu. Dans l'esprit d'une grande partie de la population israélienne, la construction de cet édifice a atteint son but, le conflit a été déplacé « de l'autre côté du mur ».
\end{abstract}

Toutefois, si le nombre d'attentats a chuté et si les interactions avec des Palestiniens relèvent de l'exception, la séparation des deux territoires est en revanche loin d'être achevée et la construction traine en longueur. A l'été 2008, soit trois ans après la date initiale prévue pour l'achèvement des travaux, moins

1. Le terme de «barrière de sécurité » (gader bitahon) est, avec celui de «barrière de séparation » (gader hafrada), l'un des termes utilisés par les Israéliens pour désigner ce dispositif de séparation. Dans cet article, j'utiliserai alternativement les termes de «barrière » et de « mur » puisque cet édifice est constitué, selon les lieux, d'un ensemble complexe de murs de béton ou de clôtures électroniques, et de routes de patrouilles.

2. Hass A., "Israel Closure Policy: An Ineffective Strategy of Containment and Repression", Journal of Palestine Studies, $\mathrm{n}^{\circ} 31$, printemps 2002, pp. 5-20. 
des deux tiers de son tracé sont accomplis ${ }^{3}$ et certaines parties ne sont pas encore opérationnelles ${ }^{4}$. Par ailleurs, la ligne de séparation qu'établit aujourd'hui la barrière entre la Cisjordanie et Israël est brouillée par le déploiement de dispositifs de contrôle (checkpoints, clôtures, tours de guet, tranchées) qui viennent renforcer la visibilité de la présence israélienne du «côté palestinien » du mur. Ils renforcent ainsi l'enclavement des agglomérations palestiniennes encerclées par des zones sous contrôle israélien, et sur une carte, ils rendent radicalement impossible la délimitation de deux territoires distincts, l'un israélien et l'autre palestinien.

Dans un contexte où le mur reste largement inachevé et où les démarcations qu'il génère sont doublées et brouillées par d'autres repères spatiaux, on peut se demander comment la grande majorité des Israéliens se représente la séparation. Cette question me semble d'autant plus importante à élucider à un moment où le projet de construction cesse d'être une priorité aux yeux des décideurs politiques et semble avoir été largement oublié par la population. A l'automne 2007, on annonce dans les journaux qu'une partie des budgets alloués à sa construction a été reportée au profit de programmes d'entraînement et de rééquipement de l'armée israélienne ${ }^{5}$. Le budget pour les années 2008 et 2009 tombe ainsi à moins des deux tiers de la somme nécessaire à sa finition, reportant à une date indéterminée l'achèvement des travaux. Au cours de l'été 2008, le colonel de réserve Shaul Arieli exprime même des doutes sur la possibilité d'achever un jour cette structure ${ }^{6}$. Tout se passe comme si, du point de vue de la population juive israélienne, la construction de la barrière n'était plus nécessaire à la restauration de la sécurité et de la souveraineté dans les zones contrôlées par Israël. En un mot, la capacité de ce mur inachevé à renforcer chez les Israéliens le sentiment d'être séparés des Palestiniens pose la question du rôle de la matérialité des frontières dans la construction sociale et politique de ces mêmes frontières.

Je propose de répondre à ces questions en plusieurs étapes. Tout d'abord, afin de comprendre comment la population juive appréhende cette séparation au-delà des discours officiels de ses représentants politiques, il convient de resituer le contexte qui prévalait en 2002 lors du lancement de la construction de la barrière. Cela permettra de comprendre à quelles attentes répond l'érection de cet édifice, à travers quels critères les gens évaluent son efficacité et comment ils envisagent la séparation. Je m'attacherai donc à mesurer l'efficacité du mur non pas au niveau des arrangements spatiaux, mais plutôt au niveau des représentations qu'il a pu faire émerger au sein de la population

3. UNOCHA, UNRWA, Four Years after the Advisory Opinion of the International Court of Justice on the Barrier, Jérusalem, UNOCHA, 2008.

4. Igal Palmor, porte-parole du ministère israélien des Affaires étrangères, entretien à Jérusalem, décembre 2007.

5. Rappaport A., “Lo Ro'im et Hasof”, Maariv, n`18281, 28 octobre 2008, pp. 2-3.

6. Harel A., "The Delays in the Fence", Haaretz, 28 juillet 2008, p. 1. 
israélienne concernant les évolutions de l'espace et du pouvoir de l'Etat. Comme l'explique Peter Andreas 7 , l'efficacité d'une politique frontalière ne se mesure pas seulement à sa capacité à empêcher le passage, mais aussi largement à son pouvoir de restauration symbolique de l'autorité et de la souveraineté de l'Etat dans les limites qu'il revendique.

C'est dans un deuxième temps que j'analyserai l'efficacité du mur à séparer concrètement la population israélienne des Palestiniens, ainsi qu'à sécuriser les zones à l'ouest de son tracé. Je montrerai combien sa capacité est largement surévaluée. Cette surestimation est favorisée par l'efficacité des dispositifs de contrôle qui fonctionnent parallèlement au mur. Moins visibles, moins médiatisés, ce sont pourtant eux qui jouent un rôle majeur dans la diminution des attentats et la réduction des interactions entre Palestiniens et Israéliens.

Je verrai enfin que si ces dispositifs sécuritaires déployés parallèlement au mur peuvent renforcer l'efficacité de celui-ci aux yeux des gens qui vivent à l'intérieur de la Ligne verte ${ }^{8}$, ils peuvent également brouiller les lignes de démarcation pour les populations vivant le long de l'édifice. Ce dernier point conduira ainsi à évaluer dans quelle mesure des techniques de " contrôle à distance » et des « régimes de mobilité » qui fonctionnent selon des logiques territoriales contradictoires ${ }^{9}$ peuvent conforter ou, au contraire, remettre en cause les représentations spatiales qui s'inscrivent dans les modèles frontaliers propres aux Etats modernes.

Mon analyse se fonde sur des données ethnographiques recueillies lorsque je résidai en Israël entre 2000 et 2004, puis entre 2007 et 2008 ; et lors de trois séjours sur le terrain, entre 2005 et 2006 . J'ai pu observer l'évolution des pratiques et des discours par rapport à la seconde Intifada chez un certain nombre de personnes rencontrées au sein de la population juive israélienne. Le but était, au départ, de comprendre quels enjeux recouvrait ce conflit à leurs yeux. J'ai ensuite écouté leurs discours et observé leurs pratiques par rapport à l'espace et je me suis demandé dans quelle mesure ils les ont modifiées, d'une part depuis la période d'Oslo (1993-2000) et, d'autre part, depuis la construction du mur. Ces personnes habitaient soit les villes de l'intérieur (Haïfa, Beer Sheva, Tel Aviv), soit des agglomérations à cheval ou proches de la Ligne verte telle que Jérusalem ou Giv'at Yeshayahu. J'ai également interviewé des colons israéliens de Cisjordanie (Bet El). Toutes ces données ont été complétées par

7. Andreas P., Border Games. Policying the US-Mexico Divide, Ithaca, London, Cornell University Press, 2001.

8. Ancienne ligne d'armistice entre les pays arabes et Israël, fixée en 1949 par le traité de Rhodes.

9. Sur les régimes de mobilité voir : Shamir R., "Without Borders? Notes on Globalization as a Mobility Regime", Sociological Theory, n⿳3 3, juin 2005. Sur les questions de contrôle à distance voir : Bigo D., Guild E. (dirs.), "La Mise à l'écart des étrangers. La logique du visa Schengen », Cultures $\mathcal{E}$ Conflits, ${ }^{\circ}{ }^{49}, 2003$. 
des observations des agencements spatiaux produits par le mur et les dispositifs de contrôle israélien. J'ai ainsi sillonné les routes croisant et longeant le mur. S'ajoutent à ces observations celles effectuées lors de mes nombreux séjours dans les enclaves palestiniennes, ce qui permet non seulement de mieux évaluer l'extension et les ramifications du régime de séparation israélien, mais également de mieux apprécier les décalages profonds au niveau des perceptions de l'espace entre les populations juives et arabes, israéliennes et palestiniennes, mais aussi au sein de la population juive.

Ces observations ne procédant pas d'une recherche systématique, mais plutôt du regard flottant d'un anthropologue, je ne prétends pas ici dresser une vision exhaustive des différentes manières d'envisager la séparation. En revanche, elles permettent d'insister sur le fait qu'en 2008, du point de vue de nombreux Israéliens, de la ville côtière de Haïfa à la colonie de Bet El, en passant par Tel Aviv et Jérusalem, le mur n'a pas encore réussi à imposer une vision claire et uniforme de la séparation entre Israéliens et Palestiniens.

\section{Le mur : support d'un imaginaire frontalier}

Avant même d'être construit, le mur a fonctionné comme le support d'un imaginaire frontalier. Sa construction n'a pas uniquement été appréhendée comme une réponse sécuritaire, mais elle a aussi été perçue comme une restauration des frontières symboliques de l'Etat et de la nation.

\section{La séparation: une quête de souveraineté et de frontière}

Pour bien comprendre cette dimension et donc ce que cet édifice a catalysé comme attentes et comme projections parmi la population juive israélienne, il faut resituer le contexte préalable à sa construction. Celle-ci intervient au moment où la population juive voit la sécurité et donc l'intégrité d'Israël menacées sur tous les fronts.

La multiplication des attentats-suicides palestiniens, dont le nombre culmine au printemps $2002{ }^{10}$, impose depuis le début de l'Intifada une atmosphère de terreur. Pendant le seul mois de mars 2002, 130 Israéliens sont tués. Outre leur dimension particulièrement meurtrière, ces attentats défient ouvertement la capacité de l'Etat à défendre ses citoyens sur son territoire au point où, comme le souligne Alain Dieckhoff, ils tendent à effacer «la frontière entre l'intérieur (le refuge du territoire national) et l'extérieur (d'où viendrait l'ennemi) 11 ». L'anxiété de la population est d'autant plus grande que l'échec du processus

10. Kershner I., Barrier. The Seam of the Israeli-Palestinian Conflict, New York, Palgrave Macmillan, 2005.

11. Dieckhoff A., «Israël : le retour du complexe de Massada », in Dieckhoff A., Leveau R., Israéliens et Palestiniens. La guerre en partage, Paris, Balland, 2003. 
d'Oslo à Camp David (juillet 2000) avait déjà donné l'impression d'aboutir à une impasse. Le fait que Bill Clinton et Ehud Barak aient accusé Yasser Arafat d'être responsable de cet échec avait convaincu les Israéliens de "l'absence de partenaire pour la Paix» et de l'impossibilité de voir un jour une résolution négociée du conflit avec les Palestiniens ${ }^{12}$.

Par ailleurs, la population juive se sent menacée sur le front intérieur. Depuis les années 1990, les leaders de la minorité palestinienne de citoyenneté israélienne remettent de plus en plus ouvertement en question le caractère juif de l'Etat, en demandant qu'Isräl devienne un "Etat de tous ses citoyens », c'est-à-dire un Etat dans lequel la minorité arabe jouirait des mêmes droits que les citoyens juifs et dont les symboles nationaux (hymne, drapeau, etc.) lui donneraient une place à part entière. Ces revendications trouvent de plus en plus de résonance dans un contexte où les négociateurs palestiniens revendiquent, de leur côté, le droit au retour en Israël - à l'intérieur de ses frontières de 1949 - pour une partie des réfugiés. Ensuite, en octobre 2000, la population juive en Israël voit ressurgir dans les manifestations des «Arabes d'Israël » le spectre tant redouté de la «cinquième colonne ${ }^{13}$ »; des manifestations qui sont d'ailleurs durement réprimées par les forces de l'ordre, faisant treize morts du côté arabe ${ }^{14}$.

Dans ce cadre, le mur n'est pas simplement envisagé comme une réponse sécuritaire et une solution unilatérale au conflit ${ }^{15}$, mais perçu également comme une solution salutaire pour le futur de l'Etat juif. Cette perception est renforcée par la campagne que mènent les leaders politiques de la gauche, parmi lesquels on trouve les principaux promoteurs du projet ${ }^{16}$. La mise en place d'une séparation unilatérale est d'abord présentée comme un moyen d'écarter Israël de la menace d'une inversion de la balance démographique en faveur des Arabes qu'aurait pu entraîner, à terme, la conservation du contrôle sur l'ensemble de la Cisjordanie et de sa population palestinienne. En outre, la séparation doit établir de manière décisive le tracé des limites d'Israël. Bien que les leaders politiques de la gauche refusent encore de se référer à la notion de «frontière » 17 , la fascination qu'exerce sur la population cette séparation est d'autant plus compréhensible que l'on replace ce projet dans le contexte historique de ce jeune Etat. Moyen de protection et de conservation, le mur est, de

12. Cypel S., Les Emmurés. La société israélienne dans l'impasse, Paris, La Découverte, 2005.

13. Les Arabes restés en Israël après le premier conflit israélo-arabe de 1947-1948 ont toujours été perçus par une grande partie de la population juive comme une minorité dangereuse qui pourrait un jour se rebeller contre l'Etat au côté des autres Palestiniens.

14. Parizot C., "Désengagements électoraux ? ", La Lettre Intergéo, n², 2006, p. 5.

15. Backmann R., Un mur en Palestine, Paris, Fayard, 2006.

16. Rabinowitz D., "Borders and their Dicontents Israel's Green Line, Arabness and Unilateral Separation”, European Studies, n¹9, 2003, pp. 2-17.

17. Halper J., "Why is Israel Really Building the Wall?", The Wall in Palestine. Facts, Testimonies, Analysis and Call to Action, PENGON/Apartheid Wall Campaign's Publication, Jérusalem, 2003, pp. 172-174 ; Arieli S., Sfard M., Homa vemehdal, Tel Aviv, Sfarey alyat hagag, Yediot Aharonot, Sfarey Hemed, 2008. 
plus, envisagé comme un moyen d'achever le projet de construction nationale en donnant à Israël les frontières tant attendues d'un Etat moderne ${ }^{18}$.

Enfin, au lendemain du 11 septembre 2001, l'érection de la barrière est perçue comme l'élévation d'une frontière que nombreux voient comme civilisationnelle : un rempart entre le «monde libre » et « l'obscurantisme » ${ }^{19}$. Dans un contexte où les Israéliens ne croient plus à une solution négociée du conflit, la séparation doit être comprise comme une déconnexion du conflit et du Moyen-Orient. Cette volonté de se déconnecter est d'ailleurs exprimée d'une autre manière dans le plan de désengagement unilatéral de Gaza réalisé en septembre 2005 et dans les termes utilisés pour désigner cette opération. En Israël, on a ainsi parlé de "déconnexion » (bitnatqut) et non pas de « désengagement » (hafradat qobot).

A l'époque, les seules réserves viennent d'une partie de la droite et de l'extrême droite. D'ailleurs, lorsqu'Ariel Sharon présente le projet au cabinet pour approbation au printemps 2002, c'est avec une certaine réticence. Il ne le fait qu'après avoir succombé à la pression populaire croissante provoquée par la multiplication des attentats-suicides palestiniens. Sharon redoute que ce projet impose une frontière qui risquerait de ghettoïser les colonies israéliennes de Cisjordanie et d'aliéner les terres considérées comme un patrimoine israélien. Il accepte de lancer la construction de cet édifice sous réserve que son tracé permette de retenir les blocs de colonies les plus importants et, ainsi, conserver un maximum d'espaces en Cisjordanie ${ }^{20}$. Cependant, même au sein de la droite, la construction du mur est progressivement envisagée comme une stratégie intéressante. En permettant d'annexer de facto $80 \%$ de la population des implantations juives de Cisjordanie à Israël, il apparaît comme un moyen de déplacer la Ligne verte et, par conséquent, de « décoloniser les colonies » pour en faire des lieux de peuplement légitime ${ }^{21}$. Au cours des élections de 2006, à travers son plan de « convergence » qui vise à organiser le retrait des colonies israéliennes à l'est du mur, Ehud Olmert, chef du parti de centre droite Kadima, présente de facto le mur comme la future frontière entre les deux Etats. Progressivement donc, l'érection de cette barrière fait converger les projets politiques de la gauche et de la droite israélienne.

\section{Signes et formes de la séparation}

Tant attendue et tant espérée, la séparation est progressivement considérée en Israël comme réalisée, alors même que le mur est loin d'être achevé. Eloignés de cet édifice et ne pouvant pas évaluer la progression de sa construction, la plu-

18. Rabinowitz D., op. cit.

19. Ibid.

20. Arieli S., Sfard M., op. cit.

21. Snegaroff C., Blum M., Qui sont les colons? Une enquête de Gaza à la Cisjordanie, Paris, Flammarion, 2005. 
part des Israéliens tiennent pour preuve de la séparation l'absence d'interactions avec les Palestiniens sur le sol israélien ou dans les Territoires occupés.

Ceci est d'autant plus aisé que la réduction des interactions entre les deux populations s'impose dès 2000-2001. En effet, compte tenu des couvre-feux et des bouclages, les ouvriers palestiniens cessent de se rendre sur les chantiers de construction, dans les restaurants et dans les exploitations agricoles israéliennes. Même ceux qui passent clandestinement, sans permis de circulation, ont du mal à trouver une place tant les employeurs redoutent de les embaucher. L'Intifada alAqsa fait ressurgir les peurs qui ont prévalu pendant la période d'Oslo, durant laquelle de nombreux Israéliens redoutaient de se faire attaquer par leurs employés. Ainsi, dans des grandes villes comme Tel-Aviv, des chantiers s'arrêtent instantanément. Certains sont encore aujourd'hui à l'abandon. Dans certains immeubles de Tel Aviv, les employés de ménage arabes sont licenciés. En outre, les Israéliens cessent d'entrer dans les villes et les villages palestiniens, comme ils le faisaient pendant Oslo, pour bénéficier de services beaucoup moins chers qu'en Israël (réparations automobiles, soins dentaires, etc.). Les attaques qui ont eu lieu en 2000 et en 2001 contre certains visiteurs ont achevé de dissuader les plus téméraires. Enfin, l'armée interdit l'entrée des citoyens israéliens dans les zones autonomes palestiniennes. Pendant cette période, même les militants politiques pacifistes qui disposaient de liens parfois étroits avec des Palestiniens ont vu ces liens se distendre puis disparaître totalement.

Par ailleurs, le nombre d'attentats n'a cessé de baisser et ce, surtout au tournant de l'année 2004-2005. En 2005, le nombre de victimes israéliennes chute à 50 personnes; en 2006, il tombe à 24, puis à 13 en 2007 22. La population israélienne reprend alors confiance. La construction de la barrière donne l'impression que le conflit a été déplacé de «l'autre côté du mur » et crée le sentiment de l'émergence d'une frontière souveraine. Ceci est d'autant plus le cas dans les petites agglomérations au nord d'Israël (Meirav, Ma'ale Gilboa) et au centre du pays (Magal, Bahan et Bat Hefer) ${ }^{23}$ (voir carte). Dans une enquête récente, Nurit Kliot ${ }^{24}$ et son équipe montrent que la construction du mur dans ces zones donne aux habitants le sentiment de se réapproprier un espace autrefois aliéné en raison des attaques et des vols commis par des Palestiniens des villages voisins. Les parents des habitants de ces agglomérations ne redoutent plus de venir leur rendre visite pendant leurs périodes de vacances. Toujours selon certains habitants, la fréquentation des chambres d'hôtes par les touristes s'accroit. D'autres se félicitent du fait que le mur joue le rôle d'une «frontière » $(g v u l)$ à «l'européenne ». Il a transformé un espace autrefois appréhendé comme un «front pionnier » (sfar), en un espace souverain.

22. Harel A., "Shin Bet data shows dramatic drop in 2007 terror fatalities", Haaretz, vol. 89, $\mathrm{n}^{\circ} 27111,1^{\mathrm{er}}$ janvier, 2008, p. 1.

23. Kliot N., Khamaisi R., Shmueli D., 'Amdot ve tfisot clapei geder hahafrada beyishouvim yehudim ve'aravim, Jérusalem, The Floersheimer Institute for Policy Studies, 2007.

24 . Ibid. 


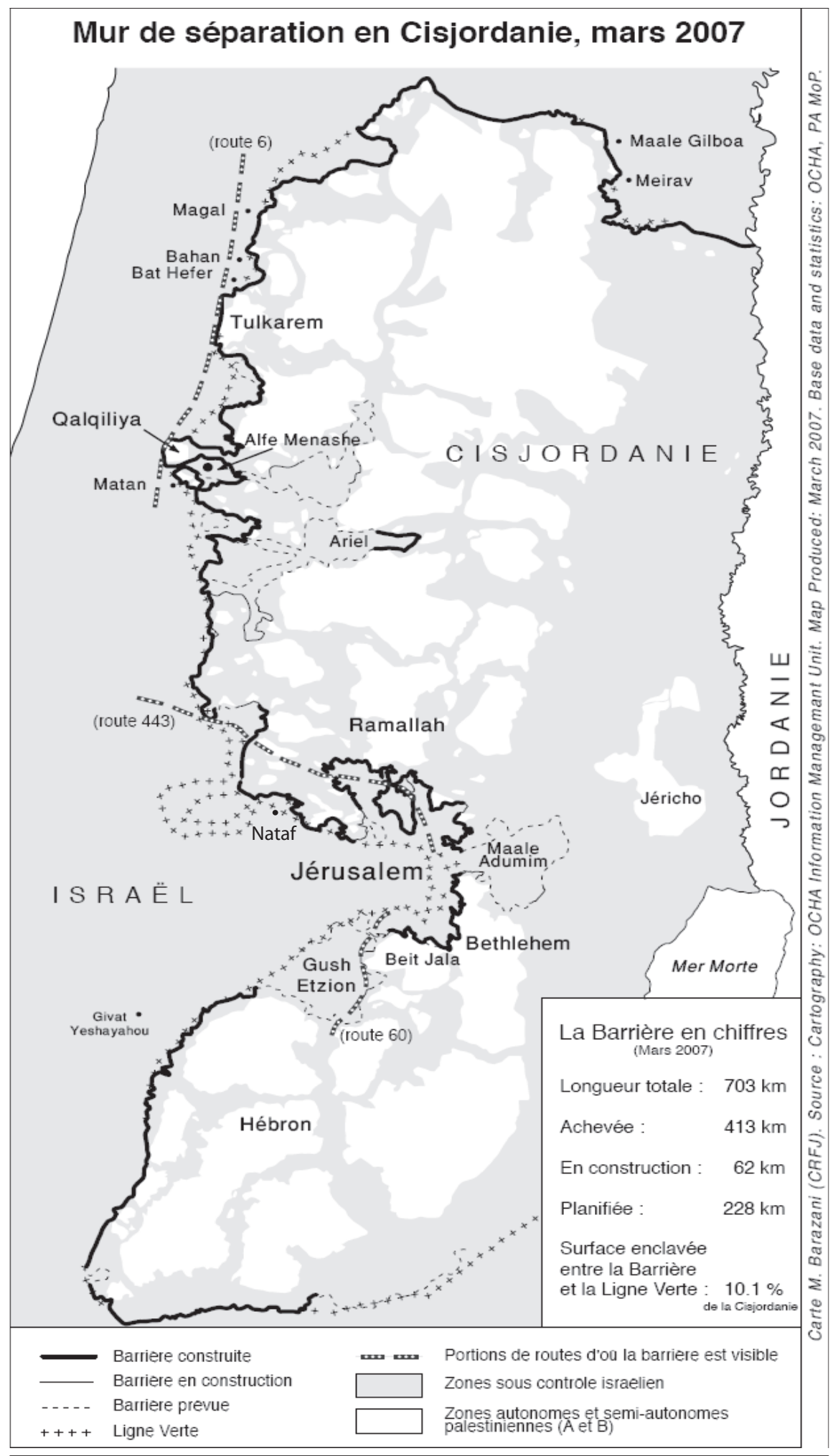


Toutefois, et de manière significative, si la grande majorité des personnes interviewées par Nurit Kliot et son équipe voient le mur comme une frontière, elles ne considèrent pas que celle-ci serve nécessairement de base aux limites du futur Etat palestinien. Ce n'est donc pas une frontière qui définit l'Autre. D’ailleurs, dans les termes employés par la majorité des Israéliens, le mur dissocie d'un côté un territoire israélien souverain et, de l'autre, les Territoires (bashtakhim) [palestiniens].

\section{Projeter la séparation}

En analysant la configuration du mur ainsi que sa place dans les dispositifs de contrôle israéliens, on peut évaluer dans quelle mesure les attentes et les images que la population projette sur cet édifice ainsi que les effets qu'elle lui attribue dépassent largement ses capacités. En ce sens, on peut dire que la capacité de séparation attribuée par la population israélienne au mur relève largement d'une projection.

\section{Projeter une continuité sur une ligne discontinue}

Cette projection est tout d'abord celle d'une continuité de type frontalière sur une ligne très discontinue. Aucun Israélien n'a fait l'expérience objective de cette continuité. Et pour cause, au cours de leurs trajets, la plupart d'entre eux ne peuvent apercevoir que quelques tronçons de cette barrière. Les personnes qui vivent près de cet édifice, dans les kibboutzim et les mochavim frontaliers, ou encore dans des agglomérations telles que Jérusalem, ne voient que des portions de mur proches de leurs habitations. De plus, ils ne croisent ces portions de mur ou de barrière que dans un nombre de lieux extrêmement limité : le long de l'autoroute trans-Israël près des villes palestiniennes de Tul Karem et Qalqilya; près du checkpoint de Kharbata sur la route 443 reliant Jérusalem à Tel Aviv, ou dans le Gush Etzion, le long de la route 60 longeant Beit Jala (voir carte). En dehors de ces zones, le réseau routier israélien ne longe pas le mur. En dehors de ces quelques lieux, les Israéliens n'ont donc pas d'endroits où ils peuvent faire l'expérience concrète de la séparation et de la délimitation spatiale entre un «ici » et un « ailleurs », entre un «chez soi » et un «chez eux ».

En outre, il est aujourd'hui impossible de faire concrètement l'expérience de cette continuité puisque la barrière reste largement inachevée. En 2008, seuls deux-tiers du tracé planifié a été réalisé, laissant encore de nombreuses ouvertures (voir carte). Incapable de répondre aux différentes pressions diplomatiques, politiques et légales, la construction de cet édifice laisse une ligne discontinue divisée en de multiples fragments. Cette discontinuité est accentuée par les «barrières de protection » (in-depth barrier) construites à l'est du mur autour des colonies juives, faisant de celles-ci des « zones extraterritoriales » au sein d'un espace palestinien. En réalité, plus le tracé du mur est ramené 
à la Ligne verte, plus le nombre de barrières de protection a augmenté pour résoudre les problèmes de sécurité, et plus le mur génère des enclaves et prend la forme d'une ligne complètement discontinue 25 .

\section{Les dispositifs de contrôle israéliens au-delà du mur}

Envisager le mur comme acteur principal de cette séparation relève d'une représentation erronée, car cela reviendrait à ignorer la présence israélienne de part et d'autre du mur.

Au cours de la période d'Oslo (1993-2000), les Israéliens ont continué à déployer des dispositifs autour et au cœur même des enclaves palestiniennes de Cisjordanie. Bien avant le mur, ils ont ainsi réduit les possibilités de séparation entre deux territoires distincts. Certes, Israël a pris une série de mesures allant dans le sens d'une séparation toujours plus systématique entre Israéliens et Palestiniens. Au fur et à mesure de la signature des accords, Isräl s'est retiré de certaines zones de Cisjordanie: des "zones $\mathrm{A}$ », où elle a délégué le contrôle civil et militaire à l'Autorité palestinienne, et partiellement des « zones $\mathrm{B}$ » pour lesquelles elle lui a délégué l'administration des affaires civiles. Elle a donc conservé le contrôle des «zones $\mathrm{C}$ », lesquelles représentent $60 \%$ de la Cisjordanie. Cependant, durant la même période, Isräl a renforcé ses politiques de bouclage et limité le nombre d'entrées des Palestiniens en Israël et dans les colonies ${ }^{26}$. Initialement articulées autour de mesures sécuritaires ad hoc, ces dispositions cherchaient à affranchir les entreprises israéliennes de la dépendance de la main-d'œuvre palestinienne ${ }^{27}$. En 2002, la volonté de séparation s'est concrétisée avec le lancement de la construction du mur. Enfin, en septembre 2005, Ariel Sharon a évacué les colons israéliens de la Bande de Gaza et a ordonné le retrait de l'armée.

Pourtant, pendant la même période, Israël a constamment renforcé son contrôle sur les zones de population palestiniennes et la violence du conflit n'a cessé de croitre. A Gaza, les Israéliens conservent le contrôle de l'espace aérien, de l'espace maritime et maintiennent le contrôle sur les flux d'importation et d'exportation. En outre, pendant cette période, les Israéliens ont continué à mener fréquemment des incursions militaires au sein de la bande côtière. Les plus massives et les plus violentes ont été celles de juin-juillet 2006 et de décembre 2008 - janvier 2009. Au lendemain du cessez-le-feu déclaré unilatéralement par Isräl le 17 janvier 2009, on compte plus de 1300 morts et plus de 5000 blessés du côté palestinien, et 13 morts du côté israélien.

25. Weizman E., Hollow Land: Israel's Architecture of Occupation, London, New York, Verso, 2007.

26. Hass A., "Israel Closure Policy: An Ineffective Strategy of Containment and Repression", Journal of Palestine Studies, n³1, printemps 2002, pp. 5-20.

27. Kemp A., Raijman R., 'Ovdim vezarim, Jérusalem/Tel Aviv, Van Leer Jerusalem Institute/Hakibbutz Hameuhad, 2008. 
En Cisjordanie, le mode de contrôle diffère. Dès les années 1990, l'Etat d'Israël a redéployé ses modes d'occupation civils et militaires et développe un réseau de près de $200 \mathrm{~km}$ de route de contournement pour les colons israéliens. Ces infrastructures ont ainsi créé un véritable maillage qui a pour effet de resserrer l'étau autour des nouvelles enclaves palestiniennes ${ }^{28}$. Au printemps 2002, au cours de l'opération « Rempart» (bomat magen), Ariel Sharon remet en cause l'intégrité de l'autonomie de ces enclaves. Il ordonne aux troupes de Tsabal de les envahir à nouveau dans le but de démanteler l'infrastructure « terroriste ». En 2002 et en 2003, les autorités israéliennes imposent des couvre-feux au sein des enclaves palestiniennes pendant plusieurs semaines, voir plusieurs mois, condamnant ainsi la population de villes entières à rester enfermée.

Si les troupes israéliennes se sont progressivement retirées de Cisjordanie depuis 2003, leur contrôle ne s'est jamais véritablement assoupli, il est même devenu de plus en plus sophistiqué.

Dans les années suivantes, afin d'accroître sa capacité à contrôler les mouvements des Palestiniens entre les diverses enclaves de Cisjordanie, l'armée y multiplie les obstacles. Elle installe des dizaines de checkpoints permanents ou volants, creuse des tranchées, érige des barrières, des blocs de bétons ou encore des monticules de terre pour barrer les routes palestiniennes. Depuis 2004, le bureau de coordination des affaires humanitaires des Nations unies estime que le nombre annuel de ces obstacles s'élève entre 500 et 600 en Cisjordanie ${ }^{29}$. Combinés à des routes de contournement (souvent) réservées aux colons et à l'armée, ces obstacles fragmentent l'espace palestinien en une multitude d'enclaves et créent ainsi une profonde distorsion de l'espace/temps entre Palestiniens et Israéliens. En se donnant la capacité de ralentir ou de bloquer la mobilité des Palestiniens, l'armée israélienne ne réduit pas seulement leur rapidité et leur liberté de déplacement, mais elle accélère aussi sa capacité d'intervention pour arrêter d'éventuels suspects ${ }^{30}$. Cette rapidité d'intervention est renforcée par la présence continue des forces armées israéliennes autour des enclaves, que ce soit dans des bases militaires, des postes avancés (checkpoints, tours de guet) ou dans les colonies de peuplement ${ }^{31}$.

La capacité d'action de l'armée au cœur des enclaves palestiniennes est enfin soutenue par les sources de renseignement dont elle dispose. Le ministère israélien de l'Intérieur conserve la gestion du registre des populations et le monopole

28. Handel A., "Where, Where to and When in the Occupied Territories? An Introduction to Geography of Disaster", in Givoni M., Hanafi S., Ophir A., Occupation: Israeli Technologies of Rule and Governance in Palestine, New York, Zone Books, à paraitre.

29. UNOCHA, West Bank and Gaza Strip Closure Maps 2007, Jérusalem, UNOCHA, 2007.

30. Ben-Ari E., Maymon M., Gazit N., Shatzberg R., From Checkpoints to Flowpoints: Sites of Friction between the Israel Defense Forces and Palestinians, Final report submitted to the Friedrich Ebert Foundation Israel, 2004.

31. Amidror (M. G. reserve) Y., Counterinsurgency War: The Israeli Experience, Jérusalem, The Jerusalem Center for Public Affairs, 2007. 
d'émission des cartes d'identité palestiniennes. Il peut ainsi suivre et affecter les trajectoires spatiales (droit de résidence dans une enclave, permis de déplacement) et sociales des Palestiniens (mariage), ainsi que de multiples aspects de leur vie quotidienne (obtention d'un permis de conduire, immatriculation de véhicule, etc.) ${ }^{32}$. Le contrôle des informations fournies par ces registres et par les réseaux d'informateurs locaux donne aux services de renseignement israéliens (Shabak) et à l'armée une grande précision pour mener ses interventions ${ }^{33}$.

Les mécanismes de contrôle israéliens cherchent à opérer une double distanciation du contrôle : dans l'espace, en le repoussant de l'autre côté du mur, et dans le temps, en contrôlant le futur grâce aux systèmes de profiling qui permettent d'arrêter les suspects avant qu'ils ne commettent des attaques contre Israël. Ces modes de " police à distance » ne renvoient pas seulement à un déplacement $\mathrm{du}$ contrôle au-delà de frontières physiques définies. Comme en Europe, ils relèvent surtout d'un glissement du contrôle de la souveraineté territoriale vers celui des identités nationales et sociales ainsi que vers les flux de personnes ${ }^{34}$.

\section{Des perceptions territoriales modernes soutenues par des formes de contrôle post-modernes}

Dans ce contexte, il apparaît non seulement que le mur n'est pas décisif dans le dispositif sécuritaire israélien, mais surtout que la séparation qu'il prétend imposer n'est possible que parce qu'elle est déjà organisée par d'autres dispositifs et mécanismes qui ont précédé sa construction. L'anthropologue Nadia Abu Zahra 35 l'avait souligné à propos du régime de mobilité imposé aux Palestiniens depuis la période d'Oslo. Elle montrait que la séparation dessinée par le mur n'est qu'une matérialisation de limites déjà imposées aux Palestiniens et à la communauté internationale par les restrictions de mouvement reposant sur la maîtrise du registre des populations.

« Le mur est venu en dernier, et non en premier. C'est la mesure finale d'une série, l'enveloppe d'une structure qui a été développée au cours de décennies. Savoir si cette séquence a été une stratégie militaire israélienne intentionnelle n'est pas ici la question. Le point est que le mur n'aurait pu être construit aussi facilement sans que des systèmes de contrôle aient été précédemment imposés à la population et étendus graduellement pour éviter un choc international et local ${ }^{36}$. »

32. Abu-Zahra N., Legal Geographies in Palestine: Identity Documentation, Dispossession, Repression and Resistance, thèse de doctorat non publiée, Oxford University, 2007.

33. Amidror (M. G. reserve) Y., op. cit.

34. Bigo D., Guild E., op. cit.

35. Abu-Zahra N., op. cit.

36. "The Wall came last, not first. It is the closing step in a series, the casing on a structure that was developed over decades. Whether or not this sequence is a purposeful Israeli military strategy is not the question here. Rather, the point being made is that the Wall could not so easily have 
D'ailleurs, dans les faits, le durcissement des restrictions de mouvements imposées aux Palestiniens n'est pas lié à la progression du mur. Selon les estimations des Nations unies, entre le premier trimestre 2001 et le premier trimestre 2002, alors que le mur n'est pas encore entamé, le nombre d'employés palestiniens se rendant en Israël et dans les colonies passe déjà de 107630 à 59 000. En revanche, du premier trimestre 2002 au premier trimestre 2007, alors que le mur est en construction, le nombre d'employés palestiniens remonte progressivement à 68 100. Ce n'est pas non plus ce nombre qui les rend invisibles aux yeux de la population israélienne. En 1996, en dehors des périodes de bouclages des Territoires occupés, les 60000 Palestiniens qui travaillaient en Israël étaient beaucoup plus visibles, dans la mesure où ils ne devaient pas se cacher comme ils le font désormais. S'ils ont aujourd'hui « disparu » du paysage israélien, c'est en raison des restrictions de mouvements plus fortes qui les criminalisent et les condamnent à la clandestinité ${ }^{37}$. Enfin, la baisse des attentats souvent attribuée à la construction du mur n'aurait pu être réalisée sans les mécanismes de contrôle déployés au-delà de ses limites ${ }^{38}$.

Finalement, en reconfigurant les espaces pratiqués par les Israéliens, sans pour autant en éloigner géographiquement les Palestiniens, ces dispositifs de contrôle renforcent la capacité du mur à produire un sentiment de séparation et de frontière entre Israël et les «shtakbim».

\section{Les limites de la séparation}

Toutefois, la séparation établie par les dispositifs de pouvoir israéliens connaît certaines limites. Si elle est efficace aux yeux des populations israéliennes vivant loin de l'édifice, c'est-à-dire dans les petites et grandes agglomérations situées sur la côte ouest ou au centre du pays, cette séparation n'est pas toujours si nettement établie du point de vue de ceux qui vivent à l'ombre du mur. Paradoxalement, plus on se rapproche du mur, moins la distinction entre les deux territoires apparait clairement.

\section{A l'ouest du mur: des espaces séparés mais pas encore pacifiés}

Si les personnes résidant dans les kibboutzim et les moshavim ${ }^{39}$ situés le long du mur se félicitent de la construction de l'édifice et du sentiment de sécurité qu'il a rétabli, elles ne semblent pas toutes considérer vivre dans un espace totalement pacifié.

been built without prior systems of control being forced upon the population and rolled out gradually to avoid international and local shock." (traduction de l'auteur). Abu Zahra, pp. 4-23.

37. Parizot C., "Tightening Closure, Securing Disorder: Israeli Closure Policies and Informal Border Economy during the Second Intifada (2000-2006)", Refugee Watch, n³1, juin 2008, pp. 66-67

38. Amidror M.G. r. Y., op. cit.

39. Les kibbutzim sont des villages collectifs, tandis que les moshav sont des villages-coopérative. 
Le dispositif sécuritaire fait peur, il intimide. Certains habitants se posent des questions sur leur désir de poursuivre leur vie dans un tel univers sécuritaire ${ }^{40}$. De même, bien qu'il sécurise l'espace pour certains résidents, le dispositif peut en revanche donner au paysage un aspect qui rebute les visiteurs venant d'autres localités d'Israël. Les visiteurs se rendant dans des agglomérations comme celle de Mattan (face au village palestinien de Habla, et non loin de la ville de Qalqiliya, tous deux séparés par un mur) associent spontanément l'édifice aux checkpoints. Les visiteurs s'interrogent sur la présence possible de ces derniers dans les environs, sur la probabilité d'avoir à passer par de tels barrages militaires ${ }^{41}$. En d'autres termes, si le mur donne aux visiteurs l'impression d'être protégés d'attaques palestiniennes, il peut, dans le même temps, leur donner l'impression d'entrer dans une colonie de peuplement située dans les Territoires occupés, alors qu'ils sont en fait dans une agglomération à l'intérieur d'Israël (dans ses frontières d'avant 1967).

Le mur peut donc également apparaitre comme un repère visuel repoussant. Ces réactions font écho aux efforts des autorités et des populations locales dans certaines régions pour le camoufler. Ainsi, sur certaines portions (Beit Jala), le mur est « habillé » de pierres agrafées sur sa surface ; à Jérusalem certaines portions sont peintes. Le long de la route $n^{\circ} 6$, près de Qalqilya, des pelouses et des arbres ont été plantés sur de grands remblais de terre.

Au-delà, certains riverains doutent également de son efficacité. A Nataf, une agglomération à l'ouest de Jérusalem, au sud de la Ligne verte et de la barrière (voir carte), les habitants se plaignent du fait que la structure n'empêche pas les voleurs palestiniens de venir cambrioler les maisons qui y sont installées. Un habitant expliquait qu'au début, ces voleurs passaient par les trous d'égouts sous la clôture, puis ensuite par des trous qu'ils découpaient euxmêmes dans le grillage. A l'été 2008, les Palestiniens du village de Qatana avaient démonté entre un et deux kilomètres de grillage (voir photos $\mathrm{n}^{\circ} 1$ et $\mathrm{n}^{\circ} 2$ ) que l'armée n'avait toujours pas refermés en janvier $2009^{42}$. Les riverains sont d'autant plus inquiets que certains d'entre eux se sont fait rançonner par des Palestiniens tandis qu'ils se promenaient près du grillage.

40. Kliot N., Khamaisi R., Shmueli D., op. cit.

41. Ibid.

42. Constat fait lors d'une visite sur le terrain entre Nataf et le village palestinien de Qatana, 5 janvier 2009. 
Figure $n^{\circ} 1$ : Vue partielle de portion de mur démontée par les habitants de Qatana

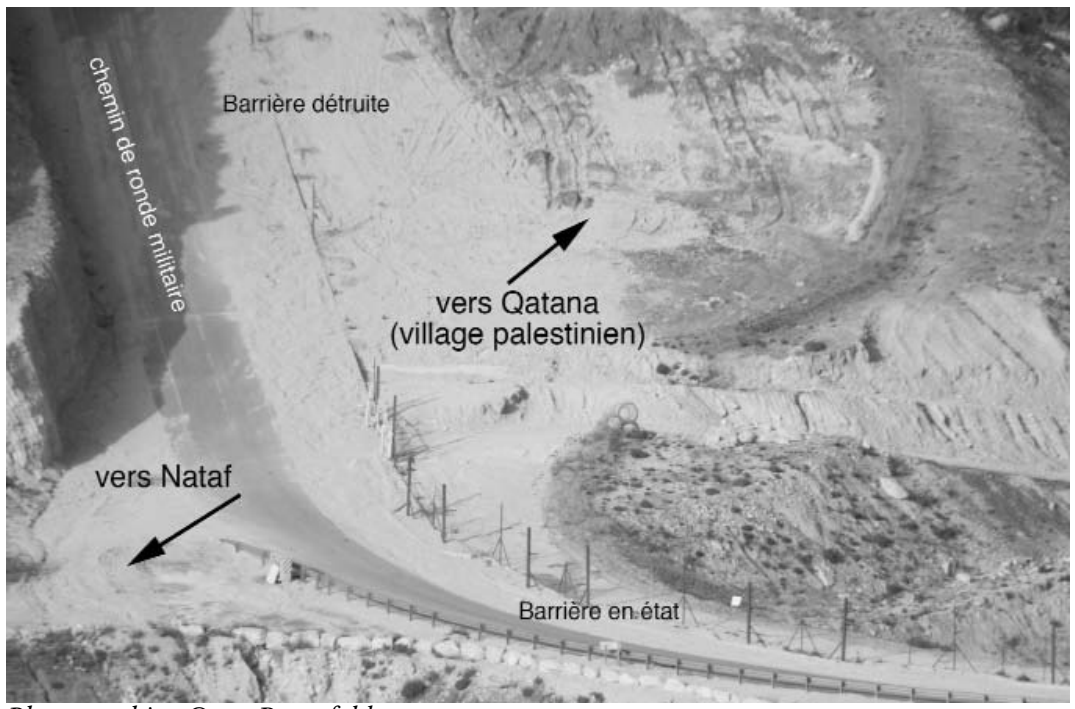

Photographie: Oren Rosenfeld

Figure $n^{\circ} 2$ : Vue globale de portion de grillage arrachée par les Palestiniens de Qatana

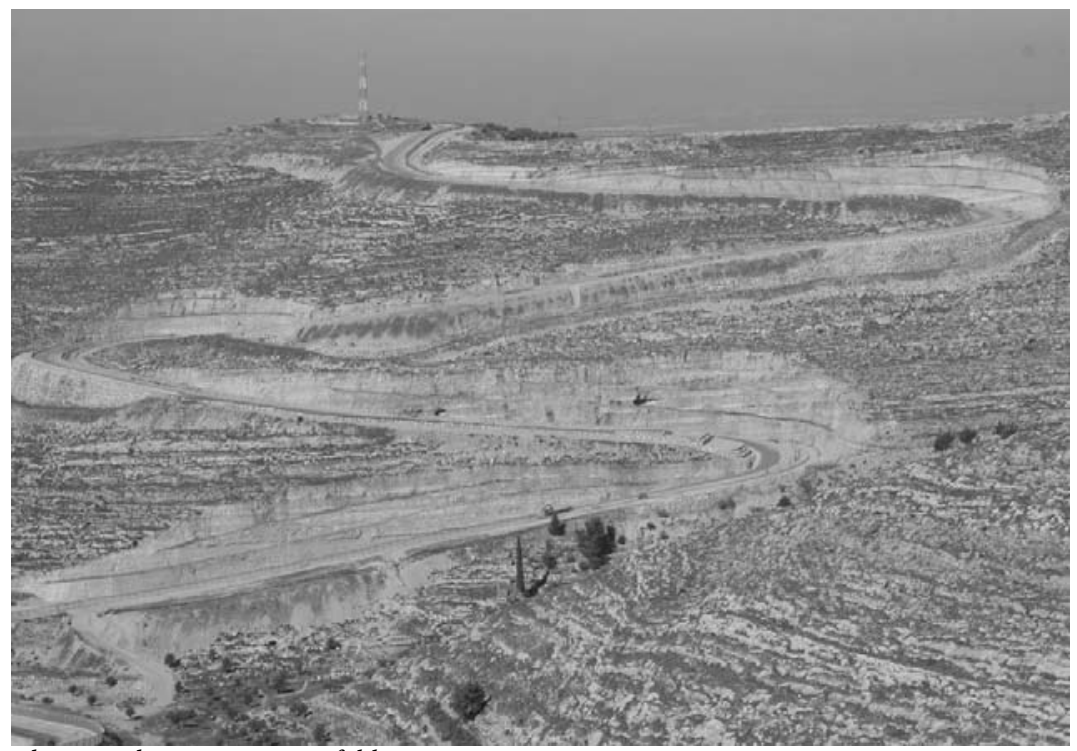

Photographie: Oren Rosenfeld

Une borne pas toujours significative

Le mur n'apparaît pas toujours comme une borne significative pour marquer la limite entre Israël et les «Territoires ». L'expérience d'un jeune couple israélien résidant dans le moshav de Giva'at Yeshayahou à 40 minutes au sudouest de Jérusalem (à l'ouest de la Ligne verte) illustre tout à fait ce point. 
Yoav et Sarah sont architectes d'intérieur. Ils se rendent fréquemment au Sud de Jérusalem pour leur travail ou pour y faire des achats. C'est le cas, notamment, quand ils vont dans le quartier de Talpiot pour faire leurs courses dans l'un des supermarchés de Jérusalem. Pour y aller, ils passent par le Gush Etzion ${ }^{43}$ et par la route des tunnels (voir carte). Ils traversent la Ligne verte avant le village palestinien de Husan et la colonie israélienne de Beitar Ilit. Ils récupèrent ensuite la route 60 réservée aux Israéliens et longent un mur de 8 mètres de haut pour arriver au checkpoint marquant l'entrée vers la zone de Jérusalem (voir photo $\mathrm{n}^{\circ} 3$ ). Ils empruntent ensuite les tunnels qui passent sous la ville palestinienne de Beit Jala pour rejoindre le sud de Jérusalem. Bien qu'étant à la périphérie de la Cisjordanie et demeurant du côté ouest du mur, ils me disent avoir l'impression de passer au cœur des Territoires occupés. En revanche, lorsque Yoav s'est rendu sur des chantiers à Mishor Adumim, une colonie israélienne à l'est de Ma'ale Adumim, ou sur la Mer morte avec sa femme, son expérience a été tout à fait différente. Lors de ces trajets, Yaov et Sarah passent un checkpoint, puis se rendent de l'autre côté du mur pour s'enfoncer au cour de la Cisjordanie. Cette fois pourtant, ils n'ont pas la même impression d'évoluer dans les Territoires occupés.

Ils expliquent que la différence entre les deux parcours tient à la présence des Palestiniens et à celle des dispositifs sécuritaires très intimidants. En passant par le Gush Etzion pour se rendre à Jérusalem, Yoav me dit croiser de nombreux véhicules avec des plaques vertes ${ }^{44}$ et de nombreux Palestiniens sur le bord des routes. Il est enfin particulièrement impressionné par les murs de bétons dont la partie supérieure est inclinée (voir photo n ${ }^{\circ} 3$ ). Il ajoute : «Ces murs n'ont pas été mis là pour rien! ». Il se sent d'autant plus menacé qu'il dit savoir qu'au début de la seconde Intifada, plusieurs personnes sont mortes sur cette route, tuées au volant de leurs véhicules par des snipers palestiniens. Mais sur la route de la Mer morte, il se sent loin des Palestiniens. Il dit n'en croiser presqu'aucun en dehors de quelques Bédouins. Et pour cause, la route de contournement construite pendant le processus d'Oslo a permis aux Israéliens se dirigeant vers Ma'ale Adumim et la Mer morte d'éviter la ville palestinienne de 'Azariyya mais, surtout, d'éloigner visuellement les Palestiniens 45.

Ce n'est pas ici la localisation du mur qui permet à ce couple de se situer dans l'espace mais plutôt les autres repères qu'ils croisent. Entre Jérusalem, Maale Adumim et la Mer morte la qualité de la route à quatre voies dont la construction a été entamée en 2003, la présence exclusive d'habitations israéliennes, l'absence de Palestiniens et la discrétion des dispositifs sécuritaires

43. Le Gush Etzion est un bloc de colonie de peuplement au sud-est de Jérusalem. A l'instar de Ma'ale Adumim et Ariel, il est considéré par les Israéliens comme l'un des principaux blocs qui devrait être annexé à Israël dans le cadre d'une résolution du conflit.

44. Depuis la période d'Oslo (1994-2000), les voitures palestiniennes sont immatriculées avec des plaques vertes (lettres vertes sur fond blanc ou lettres blanches sur fond vert), alors que les voitures israéliennes ont des plaques de couleur jaune.

45. Snegaroff C., Blum M., op. cit. 
Figure $\mathrm{n}^{\circ} 3$ : Murs de béton inclinés séparant la route soixante de Beit Jala, 27 mai 2007

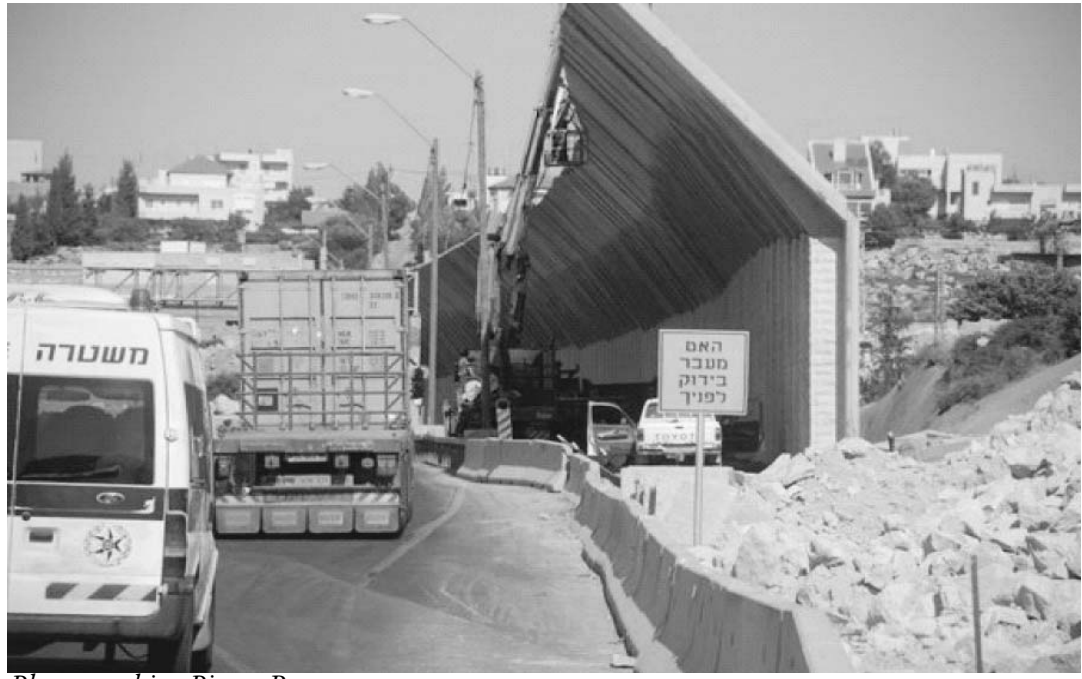

Photographie : Pierre Renno pas suffisante pour le pacifier complètement et donc pour l'« israéliser ».

Les repères rencontrés fonctionnent donc comme des bornes qui établissent une continuité israélienne le long des trajectoires spatiales de ce couple. Ces bornes mettent également en scène un rapport de force. Car si l'espace est mixte, il reste néanmoins dominé et donc sécurisé par les autorités israéliennes. Nous sommes donc ici dans une autre approche territoriale que celle définie par le mur. L'érection d'une barrière, nous l'avons vu, renvoie à la territorialité de l'Etat moderne en définissant un espace qu'elle borne sur ses contours. Cependant, le rapport à l'espace de ce couple et des personnes qui évoluent dans ces lieux proches du mur tend à se construire sur les repères qui sont au centre, et non sur les extrémités, de l'espace. On cerne donc ici une double limite du mur à définir la nature des lieux traversés. Non seulement, il n'est pas systématiquement envisagé comme une frontière pertinente, mais, en outre, il n'arrive pas à imposer les réflexes territoriaux auxquels il renvoie.

\section{Une décolonisation imparfaite}

La difficulté du mur et des autres dispositifs israéliens à donner le sentiment aux Israéliens d'être complètement séparés des Palestiniens est d'autant plus flagrante à mesure que l'on évolue entre la Ligne verte et l'ouest du tracé du mur. Elle montre l'échec relatif de cette structure à « décoloniser » totalement les colonies de peuplement israéliennes à l'ouest de l'édifice. Les habi- 
tants de ces agglomérations espéraient pourtant beaucoup de la construction de cet édifice. Influencer les décideurs politiques et les planificateurs pour se trouver « du bon côté de la barrière » était envisagé comme un moyen de sauver une colonie du dépeuplement voire assurer sa prospérité.

Si à Alfei Menashe, une colonie au nord-est de Tel Aviv, le mur a rassuré les habitants, nombre de leurs parents ne vont pas leur rendre visite en raison du paysage qui l'entoure. En 2003, la construction de la barrière a permis d'opérer une normalisation et une reprise de la croissance de cette colonie de 6000 habitants. Les constructions de maisons ont repris et les prix des logements ont considérablement augmenté. De ce point de vue, la Ligne verte s'est progressivement effacée ${ }^{46}$. Néanmoins, la présence de villages palestiniens enclavés ('Arab ar-Ramadhîn al-Janûbi, Khirbet ad-Daba et Râs at-Tîra), ainsi que les barbelés, les routes de patrouilles et les checkpoints viennent rappeler à certains le passage de la Ligne verte. Pour ces personnes, ces signes importent encore davantage que le mur. Ainsi, certains résidents des villes de Ra'anana et de Tel Aviv dont des membres de leur famille vivent à Alfei Menashe ont dit refuser d'aller voir leurs parents car ils redoutaient d'entrer dans ce qu'ils appelaient les «shtakhim».

Dans la colonie d'Ariel, ce sont les colons eux-mêmes qui se trouvent de plus en plus affectés. Tout en étant du côté ouest du mur, la population de cette colonie voit ses conditions de vie devenir de plus en plus contraignantes. Les dispositifs de contrôle (checkpoints, grillages, etc.) déployés pour assurer leur protection effrayent de nombreux visiteurs et accentuent leur isolement social ${ }^{47}$. Ils gênent également les déplacements des colons. Les checkpoints créent des embouteillages fréquents qui rallongent les temps de déplacements et éloignent les habitants du centre du pays. Les frustrations générées par ces changements de conditions de vie sont telles que le maire d'Alfei Menashe expliquait dans une interview accordée au journal Haaretz qu'il craignait que, si une compensation était offerte aux habitants des colonies se trouvant à l'est du mur, une grande partie de la population d'Ariel la demanderait aussi.

Le projet de construction du mur et les tronçons réalisés jusqu'à présent fonctionnent comme des supports qui ont permis à une grande partie de la population juive israélienne de projeter de multiples attentes et représentations au point d'envisager la séparation comme donnée. Il est d'autant plus pertinent de parler de «projection de représentation » dans la mesure où, bien avant sa construction, le mur jouait déjà le rôle d'un tel support. De plus, six

46. Ibid.

47. Eldar A., "Border Control: Settlers for Sale”, Haaretz, vol. 89, n²6911, 4 décember 2007, p. 7. 
ans après le début des travaux, alors que moins des deux tiers du tracé ont été achevés, les Israéliens attribuent à cet édifice une continuité qu'il n'a pas. En effet, tout d'abord la majorité des Israéliens ne croisent pas et donc ne voient pas sa route. Ils ne peuvent donc pas faire l'expérience de son caractère discontinu, de l'inopérabilité de certaines portions, ou encore des nombreuses ouvertures qu'il reste à combler ou même à réparer. Enfin, la population israélienne attribue au mur des fonctions qui sont en réalité largement assurées par des mécanismes de contrôle moins visibles et moins médiatisés.

A ce niveau, il est possible d'apprécier la capacité des mécanismes de contrôle inscrits dans des formes de territorialités post-modernes (régime de mobilité, police à distance, etc.) à renforcer les perceptions territoriales frontalières modernes parmi la population de l'Etat qui déploie ces mêmes mécanismes. Organisant la séparation des mouvements entre les populations israéliennes et palestiniennes, opérant une surveillance très stricte des groupements armés palestiniens, ce sont ces mécanismes qui, bien avant l'achèvement du mur, renforcent le sentiment des Israéliens d'être séparés des Palestiniens. Ayant fait chuter les attentats et ayant restauré la souveraineté de l'Etat, ils donnent l'impression qu'une séparation de type frontalière a été établie entre Israël et les «shtakhim».

Mais on ne saurait exagérer la capacité de ces dispositifs de contrôle. Les infrastructures et les réglementations sur lesquelles reposent ces dispositifs peuvent également brouiller les cartes. Paradoxalement, plus on se rapproche du mur, plus la séparation qu'il est censé établir perd de sa netteté. Les checkpoints, les barrières, les monticules de terres, mais aussi toutes les règles qui s'imposent aux colons israéliens pour assurer leur sécurité viennent saturer l'espace au point de devenir des repères plus voyants et plus significatifs que le mur. Dans des régions comme le Gush Etzion, la barrière n'arrive pas à normaliser l'espace annexé de facto par Israël. Tout dépend donc de la visibilité de ces dispositifs.

Ceci conduit à relativiser le rôle de la matérialité des frontières dans la construction de ces mêmes frontières. Du point de vue de la majorité des Israéliens qui se trouvent éloignés de l'édifice, c'est-à-dire dans les agglomérations le long de la côte et à l'intérieur du pays, le projet de construction de mur permet de renforcer le sentiment de souveraineté, de séparation et de frontière. Inversement, du point de vue des populations israéliennes, moins nombreuses, qui résident proches du mur ou qui se déplacent le long de son tracé, celuici peut souvent perdre son pouvoir de délimitation et de marquage spatial. Il semble donc que le mur n'ait de capacité à établir une frontière que si prévaut une méconnaissance de son tracé et de sa configuration. Le mur joue ainsi un rôle plus grand en tant que projet qu'en tant que structure matérielle. En tant que projet, il fait converger les agendas politiques de la droite et de la gauche, rassure la population israélienne en donnant le sentiment de restaurer la sou- 
veraineté de l'Etat. En revanche, en tant que structure, il a du mal à imposer une vision uniforme des espaces et des frontières.

Finalement, on peut d'autant plus remettre en cause le rôle du mur à délimiter une frontière que sa construction creuse le fossé au niveau des perceptions du conflit entre Israéliens et Palestiniens. Du côté palestinien, sa présence et sa matérialité s'imposent avec la force et la violence des confiscations et de l'enfermement qu'il crée. Il donne ainsi un caractère encore plus présent et contraignant à l'occupation. Du côté israélien, son manque de visibilité pour la majorité et les projections qu'il favorise tendent à donner l'illusion d'un éloignement du conflit. En d'autres termes, renforçant le sentiment de séparation au sein de la population israélienne, le projet de construction du mur repousse, du point de vue de celle-ci, la nécessité de résoudre le conflit et de négocier une frontière entre Israéliens et Palestiniens. 